A pre and post course self-assessment of knowledge skills and confidence

Post workshop evaluations

An end of programme questionnaire

This poster describes the analysis of these.

Results The programme met the needs and expectations of $100 \%$ of participants. $100 \%$ found the education clear and felt well supported by the EOlc facilitators. Evidence shows that each cohort improved in knowledge, skills and confidence across all topics when measured on a 5-point self-assessment scale. Qualitative analysis of feedback indicates that the care homes are working as a team to utilise end of life care tools and improve patient experience approaching end of life.

Conclusion The Six Steps to Success Programme has enabled care homes to bring about organisational change and develop frameworks to improve End of Life Care in their place of work. By working together, sharing experiences and ideas, supported by their facilitators and the local hospice (Queenscourt Hospice), care homes have developed comprehensive end of life care policies which have enhanced delivery of high quality care. By rolling out the programme to a large number of care homes over a 12 month period we are creating a ground swell of enthusiastic motivated care home staff with a desire to collaborate with those working in other health and social care settings to improve the experiences of their residents and relatives.

\section{P7 RED ENVELOPE SCHEME}

Shirley Young, Deb Hickey. St Luke's Hospice, Basildon, UK

\subsection{6/bmjspcare-2013-000591.29}

Ambulance crews reported lack of information with regards to patient care $\&$ wishes, meaning their default position was Hospital admission as they could not 'waste time' looking for relevant documentation eg Do Not Attempt Cardio Pulmonary Resuscitation (DNACPR) forms, 'hidden' in copious patient records.

Aims To give crews readily accessible Patient information, enabling them to make decisions about best care, recognising patient choice.

To prevent unnecessary or unwanted hospital/hospice admissions for palliative patients. A form was designed which included Information regarding patients' Preferred Priority of Care, DNACPR, medications and contact details of relevant Health Professionals. This was agreed by the Ambulance Service.

An A5 Red envelope labelled 'FOR AMBULANCE CREW ONLY' held the completed form, to be The Scheme was piloted for 3 months from April - July 2012

During the trial 24 forms were completed and held by the patient and family

The checklist was described as 'Directional Documentation' by the Ambulance service. The contact numbers and information gave crews the opportunity to call and discuss the situation with other professionals.

Subsequent improved communication between services has resulted in Ambulance crews anecdotally describing a change in practice and increased confidence in making decisions to support patients to remain at home.

Discussion In the absence of Electronic Registers within our locality, the intermediate solution was to introduce the Red Envelope Scheme which gave Ambulance crews the right information at the right time. However despite the large catchment area of the Hospice the Scheme reaches a small percentage of patients with palliative care needs.

Conclusion Crews now look for a red envelope in the patient's home. It gives support to crews to make the right clinical decisions and the opportunity to influence the patient journey in a more positive way.

\section{P8 CARE ACROSS SETTINGS: EVALUATING AN INTEGRATED SPECIALIST NURSE-LED PALLIATIVE CARE CLINIC WITHIN A GENERAL PRACTICE SETTING}

${ }^{1}$ Emily Harrop, ${ }^{1}$ Jessica Baillie, ${ }^{1,2}$ Anthony Byrne, ${ }^{3}$ Karen Stephens, ${ }^{1}$ Annmarie Nelson. ${ }^{1}$ Marie Curie Palliative Care Research Centre, Cardiff University, Cardiff, Wales, ${ }^{2}$ Cardiff and Vale University Health Board, Cardiff, Wales, ${ }^{3}$ Marie Curie Hospice Cardiff and the Vale, Penarth Wales

\subsection{6/bmjspcare-2013-000591.30}

Background A community nurse-led palliative care clinic was introduced at a UK General Practitioner's (GP) practice in April 2012. The clinic aimed to provide earlier identification of patients with cancer and non-malignant disease, initial patient assessment, education for GPs and other community professionals, and strengthen relationships with the local multi-disciplinary team. This provides a new model for engaging patients and ensuring that specialist palliative care is delivered across different clinical settings. Usually, clinical nurse specialists (CNS) support patients in the home. This new initiative brings a fortnightly clinic as a complementary resource to consolidate the joint primary/ palliative care approach.

Methods This study evaluates implementation processes and the impact of the clinic on patient experiences and professional practices. It assesses uptake of palliative services and the management and delivery of palliative care by GPs and CNS.

Baseline interviews with GPs $(n=5)$ and CNS $(n=4)$ were carried out in February 2012. Semi-structured interviews with patients $(n=6)$, GPs $(n=6)$ and CNS $(n=4)$ are being undertaken to assess perceptions of the clinic.

Patient interviews will be analysed using an Interpretive Phenomenological Analysis (IPA) approach. Interviews with GPs and CNS will be analysed using Framework Analysis.

Results Perceived aspirations for the clinic included: earlier referral; improved communication and continuity of care; psychological and practical benefits for patients/ carers; more targeted use of specialist nursing resources. Baseline interview data highlights the need for improved referral and communication practices, especially for non-cancer patients. Data from patient and follow-up interviews will be reported against these aspirations, with additional themes identified.

Conclusions and application Conclusions will be drawn on the effectiveness of the clinic and implications identified for palliative care provision in primary care settings, including the potential to roll out a similar model more widely.

\section{P9 IS THERE ANYBODY THERE - TELEPHONE ADVICE LINES WHO NEEDS THEM?}

Tracy Livingstone. Nightingale House Hospice, Wrexham, UK

10.1136/bmjspcare-2013-000591.31

Background and Context Nightingale House Hospice have offered a 24-hour telephone advice line to patients and their carers in North East Wales since the year 2000. Predominantly 